\title{
Elective Appendicovesicostomy in Association with Monfort Abdominoplasty in the Treatment of Prune Belly Syndrome
}

\author{
Riberto Liguori, Ubirajara Barroso Jr, Joao T. Matos, Sergio L. Ottoni, Gilmar Garrone, \\ Guilherme T. Demarchi, Valdemar Ortiz, Antonio Macedo Jr
}

Division of Urology, Federal University of Sao Paulo (UNIFESP), Sao Paulo, and Division of Urology, Federal University of Bahia (UFBA), Bahia, Brazil

\begin{abstract}
Objective: To evaluate the role of elective appendicovesicostomy in association with Monfort abdominoplasty to avoid urinary tract infection (UTI) and renal damage in the post-operative follow-up of patients with prune belly syndrome. Materials and Methods: We followed 4 patients operated in our institution (UNIFESP) (Monfort, orchidopexy and Mitrofanoff) and compared them to 2 patients treated similarly, but without an appendicovesicostomy, in a second institution (UFBA). We evaluated postoperative clinical complications, UTI and preservation of renal parenchyma. Patients were followed as outpatients with urinalysis, ultrasonography (US) and occasionally with renal scintigraphy.

Results: Mean follow-up was 23.5 months. Immediate post-operative course was uneventful. We observed that only one patient with the Mitrofanoff channel persisted with UTI, while the 2 patients used as controls persisted with recurrent pyelonephritis (> 2 UTI year).

Conclusion: Our data suggest that no morbidity was added by the appendicovesicostomy to immediate postoperative surgical recovery and that this procedure may have a beneficial effect in reducing postoperative UTI events and their consequences by reducing the postvoid residuals in the early abdominoplasty follow-up. However, we recognize that the series is small and only a longer follow-up with a larger number of patients will allow us to confirm our suppositions. We could not make any statistically significant assumptions regarding differences in renal preservation due to the same limitations.
\end{abstract}

Key words: bladder; prune belly syndrome; surgical procedures, operative; urinary tract infections Int Braz J Urol. 2006; 32: 689-96

\section{INTRODUCTION}

Prune belly syndrome occurs once in 35,000 live births and consists of a triad of deficient abdominal wall musculature, intra-abdominal testes and dilated urinary tract. Extensive urinary tract reconstruction (cystoplasty, ureteroplasty and reimplantation) has moved up to more conservative approaches such as clean intermittent catheterization (CIC) aiming to avoid residual volumes. Abdominoplasty plays a role not only in improving cosmetics but also in ameliorating bladder and intestinal emptying.

We adopted in our institution the concept of performing elective appendicovesicostomies in association with the Monfort abdominoplasty and orchidopexy. We believe that providing an outlet channel 
adds little morbidity to the surgical procedure itself and brings a very beneficial mechanism of residual volume control. We hypothesized that by doing so we could be able to reduce the number of UTI and new scars per year in comparison to the classical strategy of watchful waiting. We believed as well that, in some case, we would be able to avoid the secondary need of urethral CIC, which can be a difficult issue due to the high sensitivity of the urethra.

We evaluated our concept of elective appendicovesicostomy in association with the Monfort abdominoplasty and orchidopexy with the classical strategy of abdominoplasty and orchidopexy only, by means of a retrospective comparative study involving 2 Brazilian institutions to answer this question.

\section{MATERIALS AND METHODS}

We reviewed the medical records of boys with prune belly syndrome treated in 2 centers from 1999 to 2004. We performed 6 Monfort abdominoplasties with patients ranging from 1 to 7 years (mean 3.5 years) at surgery. Antenatal diagnoses were possible in three patients and a fetal obstetric procedure was attempted in two cases (1 bladder punction and 1 vesicoamniotic shunt). One patient developed initial respiratory distress while the others were born without other complications.

Urological investigation at the time of treatment identified bladders with increased capacity and hypotonic detrusor function. Half of the patients had massive vesicoureteral reflux (VUR). All patients presented recurrent symptomatic UTI. Bowel constipation was a rule except for one patient.

The standard surgical procedure consisted of classical Monfort abdominoplasty and open orchidopexy and was conducted in 4 cases in our institution and in two cases in the associated university (Table-1). Patients operated in our center additionally received the appendix implanted in the bladder dome after removal of the urachal diverticulum (Figure-1). A cystostomy tube was left for 3 weeks and after that the patient, and family, was trained by an urotherapist nurse to perform intermittent catheterization 4 times a day (Figure-2).
Patients were followed as outpatients. They were monitored with urinalysis to check for UTI and with US for upper urinary tract evaluation. Once a year the patients underwent a voiding cystogram, renal scintigraphy and, occasionally, an urodynamic study.

\section{RESULTS}

Early postoperative course was uneventful. Patients with the Mitrofanoff channel performed CIC initially 4 times a day but subsequently were oriented

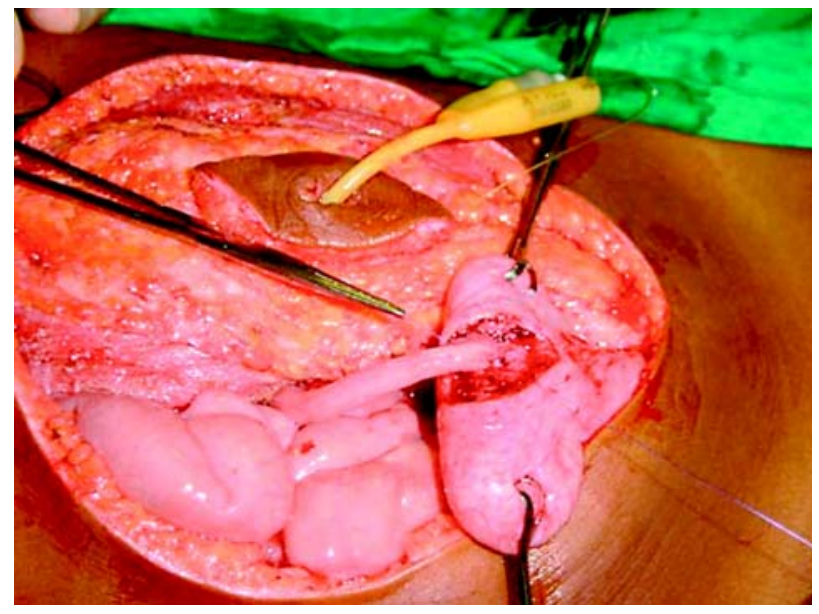

Figure 1 - The appendix being implanted in the bladder dome after removal of the urachal diverticulum.

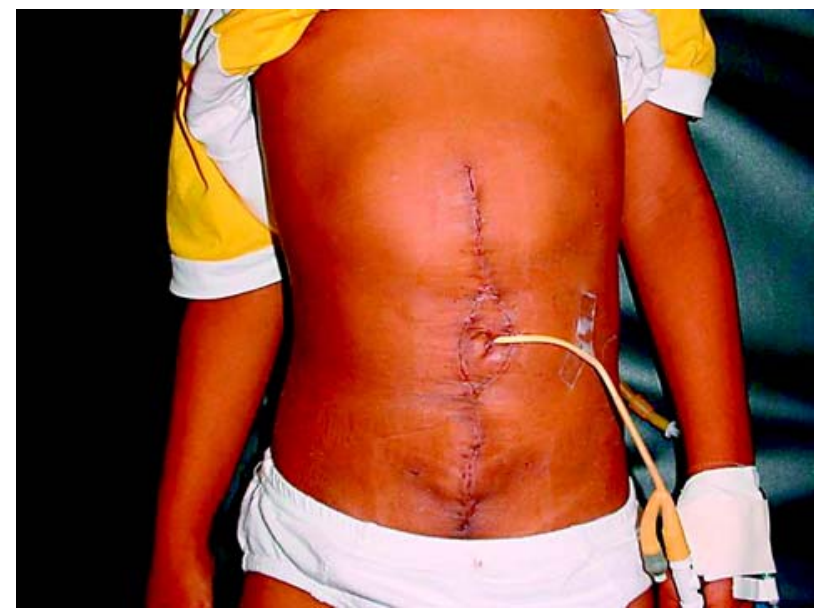

Figure 2-Abdominal wall aspect after 3 weeks of surgery. 


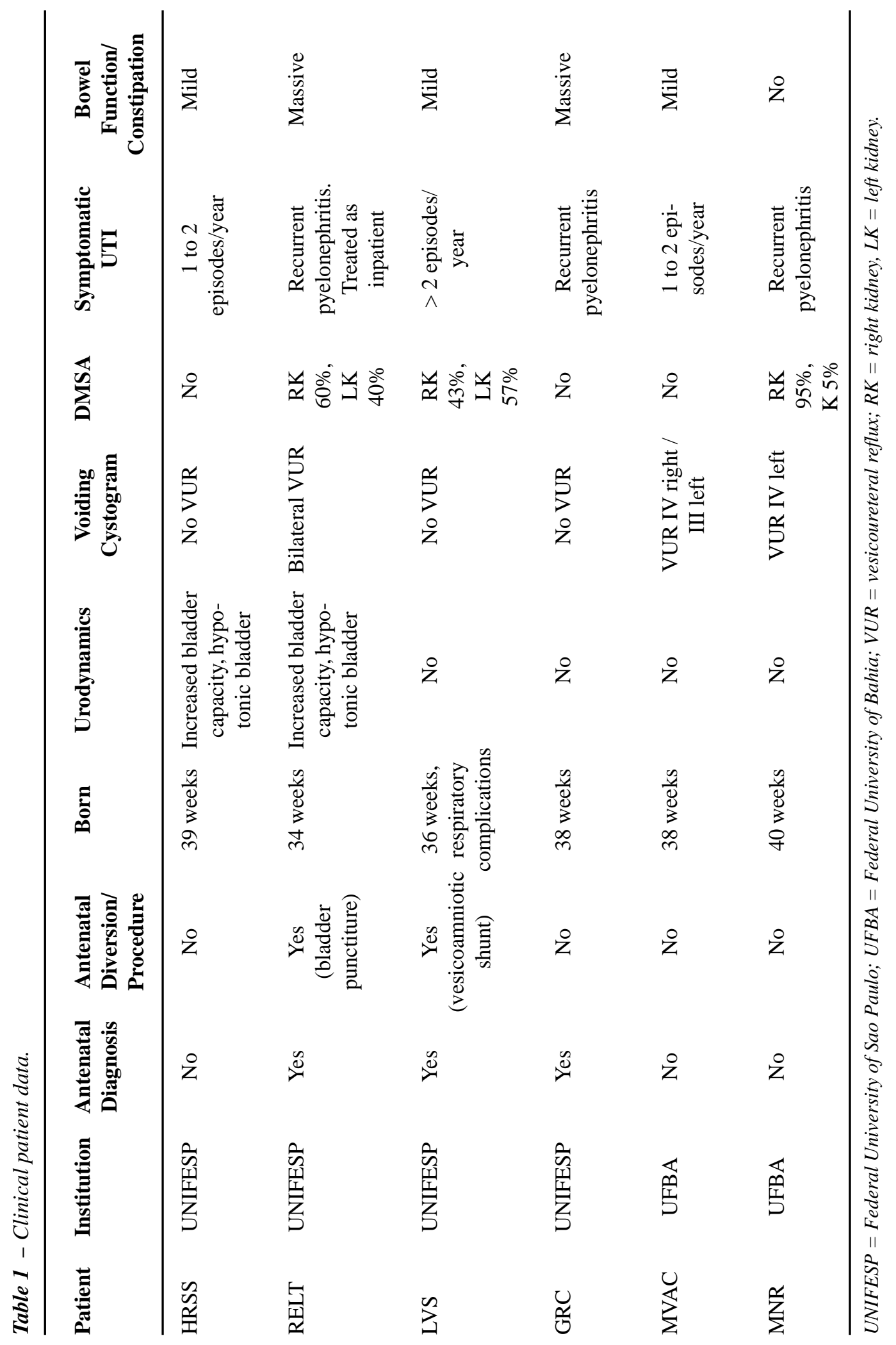


to catheterize their bladder only after a spontaneous micturition. Residual volume reduction was seen in three of four patients and CIC frequency could be reduced. Two patients had documented residual volume and therefore maintained regular catheterization over 2 times a day. One patient did not perform any CIC and another one did it only once a day. The clinical follow-up for this group of patients was satisfactory and 3 of them did not have any more clinical UTI. The patient who persisted with mild clinical UTI remained with VUR and was referred to an anti-reflux surgery.

The two patients of the other group initially maintained high residual volumes but one recovered progressively as he started voiding better. Clinically both these patients had recurrent pyelonephritis (more than 2 UTI a year).

All patients felt an improvement in constipation and were very satisfied with the final cosmetic appearance of the abdominal wall.
We could not correlate renal damage with the surgical procedure. Concerning the vesicoureteral reflux, one patient is still waiting for surgery, one has been previously operated and the other has reflux into a nonfunctional kidney and nephrectomy is being considered. Present followup is 23.5 months (Table-2).

\section{COMMENTS}

The overall prognosis of prune belly syndrome is poor, with more than $20 \%$ of extreme cases being stillborn. Renal failure will develop in approximately $30 \%$ of survivors during childhood and adolescence. Early detection of urinary infection or renal deterioration can be done by close surveillance enabling early recognition of bladder drainage abnormalities, which are the main source of complications.

Table 2 - Clinical post-operative evolution.

\begin{tabular}{|c|c|c|c|c|c|c|c|}
\hline Patient & $\begin{array}{c}\text { Age at } \\
\text { Surgery }\end{array}$ & $\begin{array}{c}\text { Elective } \\
\text { Appendicovesicostomy } \\
\text { (plus Monfort and } \\
\text { orchiopexy) }\end{array}$ & $\begin{array}{c}\text { Ureteral } \\
\text { Reimplantation }\end{array}$ & $\begin{array}{c}\text { CIC / } \\
\text { Daily } \\
\text { Frequency }\end{array}$ & $\begin{array}{l}\text { Residual } \\
\text { Volume }\end{array}$ & $\begin{array}{c}\text { Complications/ } \\
\text { Symptomatic } \\
\text { UTI }\end{array}$ & $\begin{array}{c}\text { Bowel } \\
\text { Function/ } \\
\text { Constipation }\end{array}$ \\
\hline HRSS & 7 years & Yes & No & No & No & No & $\begin{array}{c}\text { Significant } \\
\text { improvement }\end{array}$ \\
\hline RELT & 1.5 years & Yes & No & Yes / 4 & Yes & $\begin{array}{l}\text { Episodic UTI: } \\
\text { anti-reflux sur- } \\
\text { gery proposed }\end{array}$ & Improvement \\
\hline LVS & 3.2 years & Yes & No & Yes / 2 & Yes & No & $\begin{array}{c}\text { Significant } \\
\text { improvement }\end{array}$ \\
\hline GRC & 4 years & Yes & No & Yes / 1 & No & No & $\begin{array}{c}\text { Significant } \\
\text { improvement }\end{array}$ \\
\hline MVAC & 5 years & No & $\begin{array}{l}\text { Yes / Cohen } \\
\text { technique }\end{array}$ & No & No & $\begin{array}{l}\text { Recurrent pyelo- } \\
\text { nephritis / reflux } \\
\text { nephropathy / } \\
\text { hypertension }\end{array}$ & Improvement \\
\hline MNR & 1 year & No & No & No & Yes & $\begin{array}{l}\text { Recurrent pyelo- } \\
\text { nephritis }\end{array}$ & Improvement \\
\hline
\end{tabular}


Reduction cystoplasty and extensive tailoring of the ureters and reimplantation have been advocated in an attempt to improve drainage but adequate emptying can in fact be obtained with clean intermittent catheterization. The abdominal wall defect has long been regarded as a purely cosmetic disability and managed by elasticized corset-like body stocking undergarments, but clinical results with the Monfort wall plasty emphasized improvements in self-esteem, bowel function and marked reduction in post-void residual urine (1). Smith et al. reported a decrease in post-void residual volumes in 7 patients treated by the Monfort abdominoplasty without concomitant urinary tract reconstruction from $40 \%$ of bladder capacity preoperatively to $14.3 \%$ postoperatively.

Another argument in favor of reconstructing the abdominal wall is the beneficial effect on spinal stability. The prevalence of spinal deformities, particularly those related to scoliosis, in prune belly syndrome might be secondary to a chronic imbalance in spinal musculature and there is evidence that abdominal wall strengthening constitutes an important aspect in the restoration of overall trunk muscle function and stability (2).

The Monfort abdominoplasty enables an effective increase in the thickness of the anterior wall and rapidly gained popularity after its introduction to the medical community $(3,4)$.

Other techniques have been proposed as alternatives to the Monfort abdominoplasty. Furness et al. reported on an extra peritoneal plication technique which obviated the need for a fascial incision and/or entrance into the peritoneal cavity and presented adequate cosmetic results in 13 patients (5). Although this method consists of an extra peritoneal approach, only 5 patients from the series were treated without celiotomy, since most patients required at least an open orchidopexy at the time of the abdominoplasty.

We believe that the Monfort procedure is "the gold standard" technique to reconstruct the abdomen. We perform the Monfort procedure routinely for fullblown syndrome at the time of transabdominal orchidopexy in early infancy. The appendicovesicostomy procedure is a straight-forward procedure once the abdominal wall is open and the bladder prepared after removal of the urachal diverticulum. We admit, however, that controversy exists regarding the true advantage of early post-operative intermittent catheterization as defended in our study.

In our series, we were able to compare 4 patients who started urinary catheterization immediately after abdominoplasty 4 times/day through a Mitrofanoff channel with 2 patients who were not provided with an outlet channel. All patients presented recurrent UTI and bowel constipation pre-operatively. We recognize that due to the small number of patients, no definitive conclusion can be taken but we were able to identify some trends in the clinical evolution of these patients. Considering UTI as a clinical parameter, we observed that patients in the first group had significantly less UTI than patients in the second group, which had persistence of recurrent pyelonephritis (more than 2 a year). However the presence of vesicoureteral reflux is a second factor that also contributes to UTI occurrence besides post-void residuals and in cases in which both factors occur we recognize is not possible to attribute the influence of each separately. The beneficial aspects of abdominoplasty were visible in both groups, consisting of improvement in bowel habits and reduction of the residual volume. Patients with elective Mitrofanoff showed progressive reduction of residual volume and at the last follow-up only two of them needed objectively post-void catheterization (more than $40 \%$ of the capacity). In the other group, one of the two also did not present residual volume. This result suggests that abdominal wall reconstruction itself is responsible for improvement in bladder emptying and that an elective Mitrofanoff is advantageous, only during the accommodation period, for $50 \%$ of the patients and essential, for much longer periods, for the other $50 \%$.

If one considers that pyelonephritis is an important risk factor for renal scars in children less than 5 years of age, it seems logical to consider ways to minimize such risk. The popularization of CIC and catheterizable stomas in pediatric urology helped us to learn about the advantages and special cares and needs of patients and families and also about the complications of appendicovesicostomy. One could argue that when we provide a Mitrofanoff channel for every patient with prune belly we are probably overtreating many of them. This is probably true and our 
series suggests that this might have happened in half of the cases. On the other hand, the aggressive treatment of residual volume may have a role in renal function preservation although this could not be proved in this short series. We agree that the persistence of vesicoureteral reflux also might have been an important factor contributing to postoperative pyelonephritis and this should be considered when evaluating overall response to the treatment we are proposing (6).

We should note that an appendicovesicostomy is a simple surgical step when one is already reconstructing the abdominal wall but it is certainly more complicated if performed later on.

Another beneficial aspect of routine early abdominoplasty is the possibility of performing concomitant orchidopexy. Recent advances in the treatment of fertility support the idea that many patients with the prune belly syndrome may ultimately be fertile and therefore treated. Repair in infancy generally allows successful placement of the testes into the scrotum without division of the spermatic vessels which is obviously facilitated by the abdominal incision. In our series, all testes were easily brought to the scrotum and showed no evidence of retraction or shrinkage in the present follow-up.

\section{CONCLUSION}

In conclusion we believe that treatment of the prune belly syndrome is evolving and if one is interested in the functional aspects of the urinary tract our concept of early abdominal bladder emptying is appealing. As seen in this short series, no morbidity was added to the first group compared to the classical approach of abdominal wall reconstruction and orchidopexy. Our data suggests beneficial effects in reducing post-operative UTI events in the elective Mitrofanoff group of patients. We recognize, however, that the series is small and only a longer followup with larger number of patients will allow us to confirm our suppositions. We admit that this approach is novel and it is still in investigation in our Department and we recognize the opinion of others regarding the standard approach which is still abdominoplasty alone and a Mitrofanoff channel in more selected cases. However this "no risk at all strategy" with little increase of morbidity during the Monfort abdominoplasty is definitely an argument to propose this different approach.

\section{CONFLICT OF INTEREST}

None declared.

\section{REFERENCES}

1. Smith CA, Smith EA, Parrott TS, Broecker BH, Woodard JR: Voiding function in patients with the prune-belly syndrome after Monfort abdominoplasty. J Urol. 1998; 159: 1675-9.

2. Lam KS, Mehdian H: The importance of an intact abdominal musculature mechanism in maintaining spinal sagittal balance. Case illustration in prune-belly syndrome. Spine. 1999; 24: 719-22.

3. Monfort G, Guys JM, Bocciardi A, Coquet M, Chevallier D: A novel technique for reconstruction of the abdominal wall in the prune belly syndrome. J Urol. 1991; 146: 639-40.

4. Woodard JR: Prune-belly syndrome: a personal learning experience. BJU Int. 2003; 92 Suppl 1: 10-1.

5. Furness PD 3rd, Cheng EY, Franco I, Firlit CF: The prune-belly syndrome: a new and simplified technique of abdominal wall reconstruction. J Urol. 1998; 160: 1195-7; discussion 1216.

6. Liguori R, Macedo A Jr, Gonçalves I, Nobre Y, Garrone G, Hachul M, Ortiz V, Srougi M. The Monfort technique for abdominal wall reconstruction, orchidopexy and elective appendicovesicostomy in the management of the prune belly syndrome. J Urol 2005: 173; 204 (Abst 750).

Accepted after revision: September 1, 2006 


\section{EDITORIAL COMMENT}

Parallel to its main characteristics, the Prune Belly Syndrome (PBS) is also known for a variable presentation among the patients, as well as the lack of correlation between the degree of abdominal laxity and urinary tract involvement. Also, the intensity of dilatation and dysplasia of one kidney and its ureter is not the same as that of the contralateral unit. The presence of vesicoureteric reflux (VUR), and the capacity of the bladder to empty itself adequately are other variables that have to be considered when planning the treatment of these patients.

In this work, the authors present their experience with 5 patients in whom a Mitrofanoff channel was added as a means for easy catheterization, when abdominoplasty and orchiopexy were performed. The concept of intermittent bladder catheterization in PBS patients is interesting, since several, but surely not all of them, have significantly enlarged and hypotonic bladders, with post-void residuals, that are associated to urinary tract infection (UTI). Nevertheless, the presence of VUR to a dilated ureter, sometimes associated to a kidney with already limited function, is probably more important in the cause and recurrence of pyelonephritis. In the group of patients described, it seems that persistence of VUR was more important for the recurrence of UTI and pyelonephritis than the lack of the Mitrofanoff channel.

Not mentioned by the authors, the comprehensive surgical treatment, proposed by Woodard almost 30 years ago, includes the simultaneous orchiopexy and abdominoplasty with the reconstruction of the urinary tract, according to individual needs: the non-functioning kidneys and its ureters are removed, the very dilated and/or refluxing ureters are tailored and reimplanted and the very enlarged bladders are partially reduced in size, with removal of their noncontractile domes and urachal diverticulum (1). With this procedure, the anatomical conditions of the urinary tract that predispose further renal injury due to pyelonephritis are significantly reduced. In our experience of 32 patients treated comprehensively without primary diversion, 20 (including 4 without bladder reduction) had normal postoperative voiding, without residuals, and 9 presented a hypocontractile bladder, but had adequate emptying achieved with scheduled voiding associated to Credé's and Valsalva's maneuvers. Only 3 patients had significant postvoid residuals, requiring either intermittent catheterization or secondary vesicostomy. Furthermore, recurrent asymptomatic bacteriuria was observed in only 4 children, including 2 undergoing intermittent catheterization, while renal function deteriorated in only 2 patients (2).

It is our opinion that the comprehensive surgery efficiently prevents UTI and pyelonephritis in PBS patients not only by reducing urinary stasis in the bladder and ureter, but also by eliminating the VUR. The addition of a Mitrofanoff channel to the procedure, on an individual basis, may help on the long run the reduced number of patients whose bladders have significant and irreversible voiding malfunction. However, the correct preoperative identification of such patients is still matter of debate.

\section{REFERENCES}

1. Dénes FT: Surgical Treatment of Prune Belly Syndrome. In: Hohenfellner R, Fitzpatrick JM, McAninch JW (eds.), Advanced Urologic Surgery. Malden, Blackwell Publishing. 2005, pp. 458-464

2. Dénes FT, Arap MA, Giron AM, Silva FA, Arap S: Comprehensive surgical treatment of prune belly syndrome: 17 years experience with 32 patients. Urology. 2004; 64: 789-93.

Dr. Francisco T. Denes Division of Urology University of Sao Paulo, USP Sao Paulo, Brazil E-mail:f.c.denes@br2001.com.br 


\section{EDITORIAL COMMENT}

One of the most important problems related to the Prune Belly syndrome is regarding the urinary stasis and incomplete bladder emptying, both predisposing to repeating conditions of acute pyelonephritis and loss of renal function. The reduction of postvoid residuals can be obtained with reduction cystoplasty at the bladder dome, sphincterectomy, and ureteral tailoring with reimplantation, besides abdominoplasty. Clinical measures can be added such as the Valsava and Credé maneuvers and, finally, intermittent catheterization (made difficult due to the normal sensibility of the urethra). As an additional preventive measure the authors propose a systematic construction of abdominal stoma in association with the Monfort abdominoplasty and present a comparative study in 6 cases, insufficient for significant conclusions, but justifiable due to the low incidence of the syndrome. A lower incidence of pyelonephritis was obtained in the diverted group and a routine incorporation of the abdominoplasty procedure was suggested.

On reference 1 in the manuscript, the voiding function was studied in 12 patients before and after abdominoplasty. The questionnaires answered showed a subjective increase in voiding, continence, vesical plenitude sensation and urinary flow parameters. However, the urodynamic parameters, bladder capacity and maximum detrusor pressure did not change. Even though the mean residual volume dropped from $40.3 \%$ to $13 \%$, no patient required intermittent catheterization and the incidence of UTI dropped approximately $80 \%$ in all patients. Such data show a direct cause implication of abdominoplasty over the micturition quality. Dénes et al. (1) reported longitudinal abdominoplasty and urinary reconstruction in 32 patients with a mean postoperative follow up of 5 years. Twenty patients progressed with normal voiding without residual urine, 9 were compensated with Credé and Valsava maneuvers, 2 were submitted to catheterization and 1 to a vesicostomy. Renal function worsened in only 2 patients. The mentioned works included heterogeneous samples, both in relation to the severity of the cases and to the urinary reconstruction performed, making it difficult adequate comparisons. However, they suggest cutaneous derivation routinely associated to abdominoplasty, as proposed in the present work, even though with little change in morbidity, seems to mean overtreating for the majority of the patients. In the lack of a well defined criteria, the preoperative selection of cases with compromised renal functions and high residual volumes, may contribute for a more rational and precise indication of the Mitrofanoff principle. Another pertinent consideration would be the convenience of the treatment of high degree reflux together with abdominoplasty aiming at avoiding future re-intervention besides contributing to urinary infections control.

\section{REFERENCE}

1. Denes FT, Arap MA, Giron AM, Silva FAQ, Arap S: Comprehensive surgical treatment of prune-belly syndrome: 17 years' experience with 32 patients. Urology. 2004; 64: 789-94.

Dr. Paulo R. Monti Head, Section of Urology School of Medicine, Triangulo Mineiro Federal University Uberaba, Minas Gerais, Brazil E-mail:montipr@terra.com.br 